\title{
Review Paper \\ Requirements of Mental Health Services During the COVID-19 Outbreak: A Systematic Review
}

\author{
Mehrdad Kazemzadeh Atoofi, ${ }^{1,2}$, Nazila Rezaei ${ }^{3}$ (1), Farzad Kompani ${ }^{4}$ (), Fatemeh Shirzad ${ }^{1}$ (), ${ }^{*}$ Shirin Djalalinia ${ }^{3,5}$ (1)
}

1. Spiritual Health Research Center, Iran University of Medical Sciences, Tehran, Iran

2. School of Behavioral Sciences and Mental Health (Tehran Institute of Psychiatry), Iran University of Medical Sciences, Tehran, Iran.

3. Non-Communicable Diseases Research Center, Endocrinology and Metabolism Population Sciences Institute, Tehran University of Medical Sciences, Tehran, Iran.

4. Division of Hematology and Oncology, Children's Medical Center, Pediatrics Center of Excellence, Tehran University of Medical Sciences, Tehran, Iran.

5. Deputy of Research and Technology, Ministry of Health and Medical Education, Tehran, Iran.

\begin{tabular}{|l|l}
\hline $\begin{array}{c}\text { Use vour device to scan } \\
\text { and read the article online }\end{array}$ & $\begin{array}{l}\text { dftation Kazemzadeh Atoofi M, Rezaei N, Kompani F, Shirzad F, Djalalinia Sh. Requirements of Mental Health Services Dur- } \\
\text { ing the COVID-19 Outbreak: A Systematic Review. Iranian Journal of Psychiatry and Clinical Psychology. 2020; 26(3):264-279. } \\
\text { http://dx.doi.org/10.32598/ijpcp.26.2987.2 }\end{array}$ \\
dol'http://dx.doi.org/10.32598/ijpcp.26.2987.2
\end{tabular}

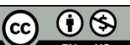

Received: 18 May 2020

Accepted: 21 Sep 2020

Available Online: 01 Dec 2020

Key words:

Covid, Coronavi-

rus, Mental health,

Intervention, Health

services, Health care

program development

\section{A B S TRACT}

Objectives After the outbreak of a new viral disease in Wuhan, China, in late December 2019, COVID-19 in a very short time and rapidly became a global pandemic. Through a systematic review, the present paper investigated the requirements of Mental Health Services during the COVID-19 outbreak. Methods A comprehensive search was conducted through PubMed and NLM Gateway (for MEDLINE), Institute of Scientific Information (ISI), and Scopus. PsychINFO and CINAHL data banks were also searched. The primary roots for the development of the search strategy developed based on the keywords of "Covid", "mental health", "care", "services". All relevant studies were included without any limitation of publication time or the papers' language as it was not possible to conduct a formal systematic review given the nature of the publications. Instead, it was decided to conduct a mixed systematic and complementary narrative review covering different interested domains.

Results Out of 80 papers, after excluding duplications, 58 articles were selected for the refinement process. Three refining steps based on the titles, abstracts, and full texts led to data extraction from 4 eligible papers. Considering the importance of the problem, related findings, key points, and research findings were summarized and presented in terms of critical components of infrastructure and resources, including policy for at-risk groups, different approaches to mental health service delivery, indirect contexts for mental health service delivery, follow-up attitudes, and complementary research. The results of the study indicate that many studies considered the consequences of physical aspects and diagnostic symptoms. Thus, aspects of mental health have been either less focused or even neglected. In mental health consequences, immediate attention and intensive programs to assess mental health, preparation for support and treatment, and prevention services are emphasized.

Conclusion During the implementation of mental health interventions, to improve services and to consider the limitations and challenges of implementing programs, it is necessary to pay attention to the attitudes of target groups and their preferences.

* Corresponding Author:

Shirin Djalalinia, PhD.

Address: Deputy of Research and Technology, Ministry of Health and Medical Education, Tehran, Iran.

Tel: +98 (915) 5120609

E-mail: shdjalalinia@gmail.com 


\section{Extended Abstract}

\section{Introduction}

evere crises, such as global epidemics, natural disasters, human-made emergencies, wars, and social crises, may cause widespread and irreparable disruptions in the areas of individual and social health. After the outbreak of a new viral disease was reported in Wuhan, China, in late December 2019, within a very short time, due to the speed of the spread, it became a global pandemic. With the ever-increasing number of people worldwide, Covid-19 has become a significant threat to the world's health and economy [1-3].

Due to the diversity and complexity, as well as the severity of the consequences related to mental health and their significant prevalence in different communities and demographic subgroups, during this period, attention to various aspects of prevention, treatment, and rehabilitation of mental health has been considered by health policymakers and researchers. Thus, studies, on the one hand, indicate the diversity of the consequences of mental health, and on the other hand, remind that due to various factors such as people's background, adaptability, coping skills, social support, and many other intervention factors of development of a single model for the prevention and management of mental disorders and crises would not be effective $[4,5]$.

Despite the importance and the urgency of the matter, it is very limited, and almost no comprehensive study has been conducted addressing the challenges to provide appropriate models and infrastructures needed for mental health services during the Covid-19 pandemic crisis [6, 7]. Therefore, it seemed necessary to evaluate and analyze the strengths and weaknesses and the most important practical points to improve mental health services provision, according to the published experiences and documents.

Based on this, the present study, using the systematic review principles and methods, discusses mental health interventions in the epidemic crisis of Covid-19 disease. This study's findings will be useful for a better understanding of the psychological impact and prospects of such a pandemic for the preparation of intervention programs to maintain and promote mental health.

\section{Methods}

To assess the Requirements of Mental Health Services during the COVID-19 all around the world. A systematic review was developed. All available related papers were searched from comprehensive international databases of PubMed and NLM Gateway (for MEDLINE), Institute of Scientific Information (ISI), and Scopus. PsychINFO and CINAHL data banks were also searched. Regardless of the study's time or the date of publication of the results and the published articles' language, all relevant reviews were included in the refinement processes.

As it was not possible to conduct a formal systematic review given the nature of the publications. Instead, it was decided to conduct a mixed systematic and complementary narrative review covering different interested domains.

\section{Quality assessment and data collection}

After conducting the searches, all of the results were saved into the Endnote software. Studies with duplicate citations were excluded. In multiple publications from the same data, the most comprehensive source of data was included (Figure 1). Remained records refined for three sequential steps of relevancy assessment, which was run according to the evaluation of titles, abstracts, and full texts, respectively. Corresponding to the study type, the quality assessment was conducted by using the standard checklists. Data were extracted from moderate and high-quality studies. Considering the scope and objectives of the study, data were extracted by a consorting checklist, information of research and its citation, target population characteristics, methodological information of analysis (the region scope, and local or survey of the study), sample size, age, sex, and exciting outcomes.

All of the mentioned procedures were conducted independently by two trained research experts. The kappa statistic for agreement on quality assessment was 0.94 . The main investigator resolved the probable discrepancy between them.

\section{Results}

After excluding duplicated papers, 58 papers were selected out of 80 papers for the refining process. Three refining steps based on the titles, abstracts, and full texts led to data extraction from 4 eligible papers. Considering the importance of the problem, related findings, key points, and research findings were summarized and presented in terms of key components of infrastructure and resources, including policy for at-risk groups, different approaches to mental health service delivery, indirect contexts for mental health service delivery, follow-up attitudes, and complementary research.

The study's main results emphasized that many studies have focused on the consequences of physical aspects and 

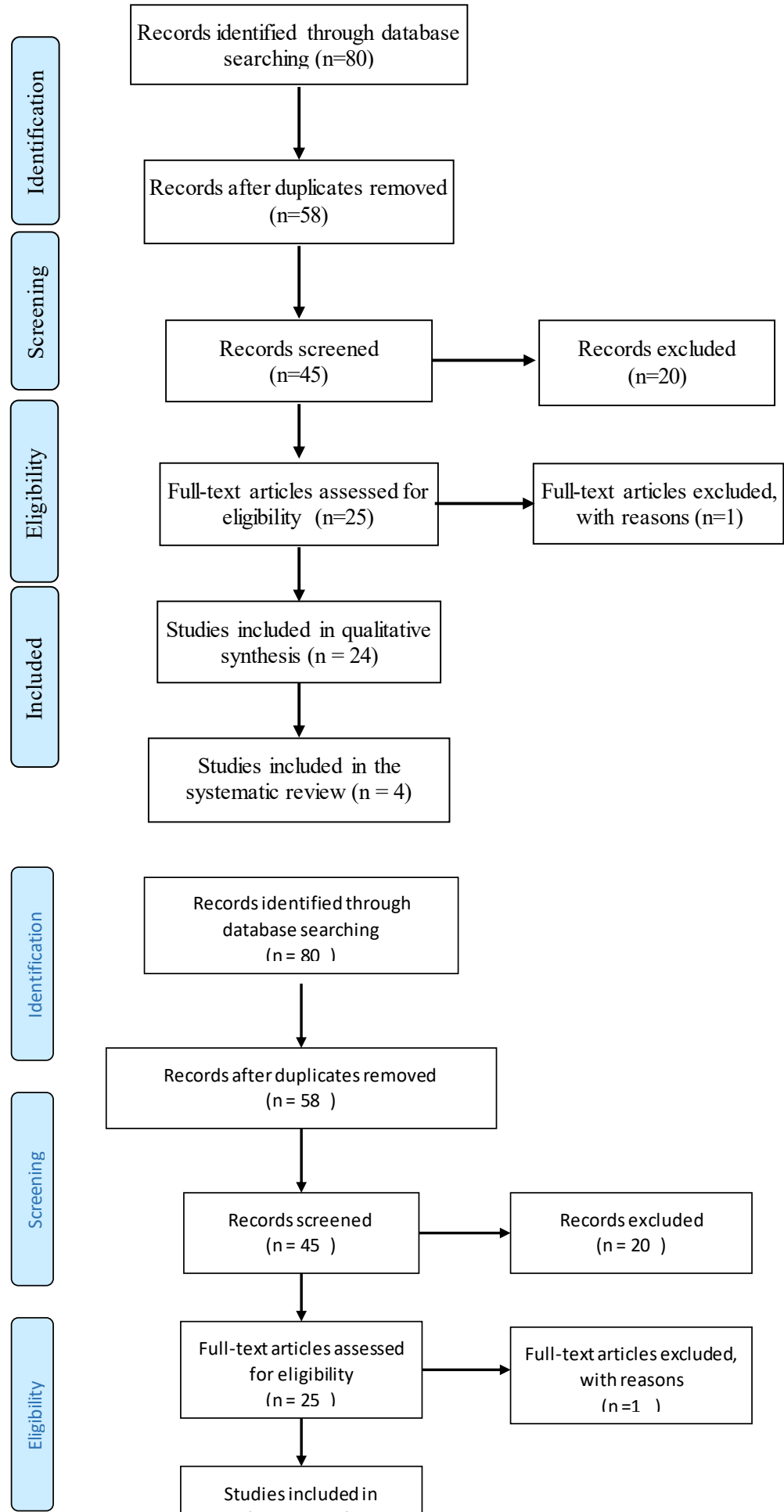

with reasons
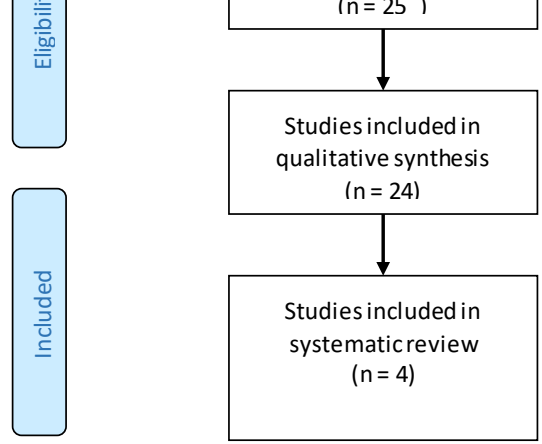

Figure 1. Papers search and review flowchart for selection of primary study 
diagnostic symptoms. In such cases, parts of mental health have been either less viewed or even neglected. In mental health consequences, immediate attention, and planning intensive programs to assess mental health, preparation for support, treatment, and prevention services are emphasized.

\section{Discussion and Conclusion}

In general, during the implementation of mental health interventions, to improve services and consider the limitations and challenges of implementing programs, it is necessary to pay attention to target groups' attitudes and preferences.

\section{Ethical Considerations}

\section{Compliance with ethical guidelines}

All included studies would be cited in all reports and all future publications.

\section{Funding}

This article is supported by Deputy of Research and Technology, Ministry of Health and Medical Education, and Spiritual Health Research Center of Iran University of Medical Sciences.

\section{Authors' contributions}

All authors contributed to preparing this paper.

\section{Conflicts of interest}

The authors declared no conflict of interest. 
This Page Intentionally Left Blank 


\title{
الزامات ارائه خدمات بهداشت روان در زمان شيوع بيمارى كوويد 9 (: يك مرور نظاممند
}

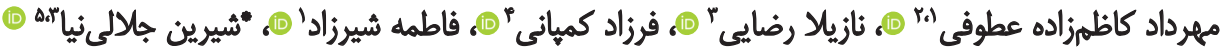 \\ ا. مركز تحقيقات سلامت معنوى، دانشكاه علوم يزشكى ايران، تهرانَ، ايران.

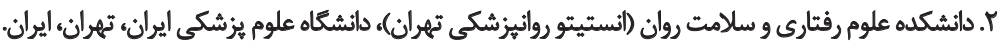

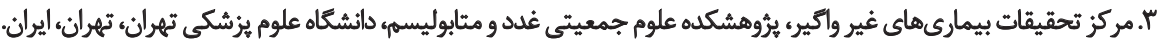

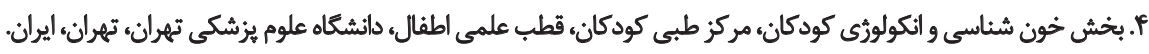

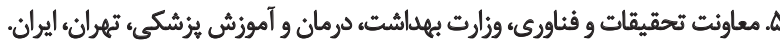

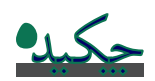

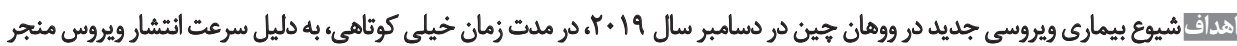

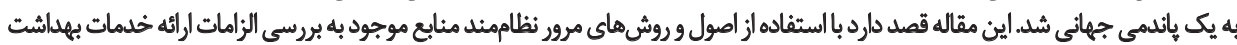

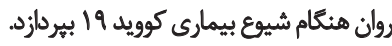

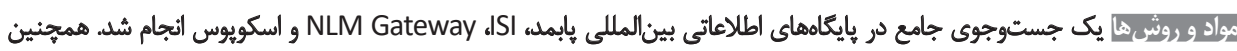

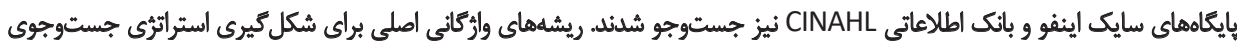

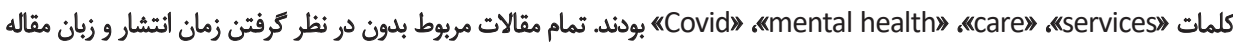

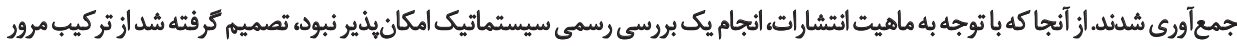

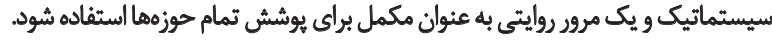

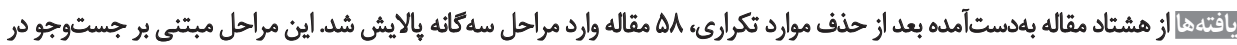

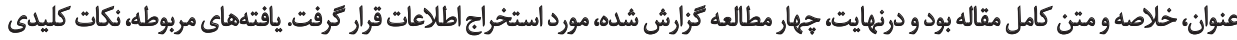

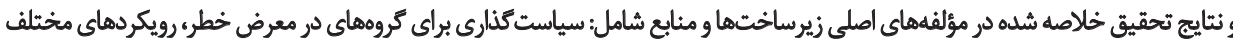

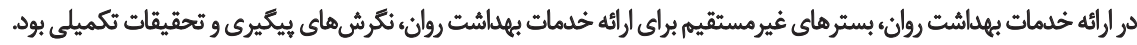

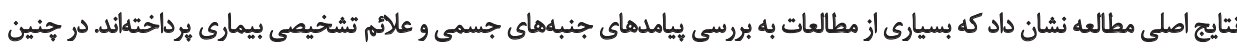

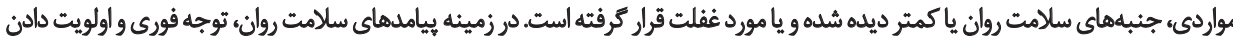

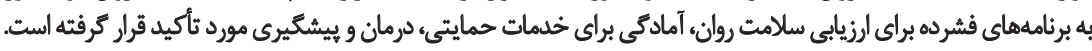

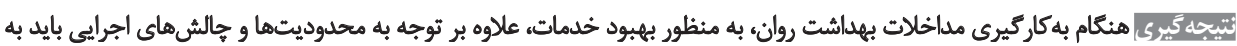

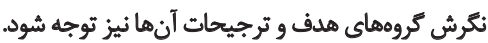

تاريخ دريافت:

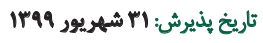
تاريخ انتشار: 11 آثر 1199

كليدوازهها:

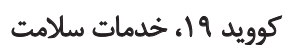

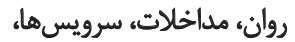
مراقبث مدان

فاصله زماني بسيار كوتاهي از ثبت و اعلام نخستين كزارشات،

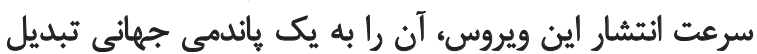

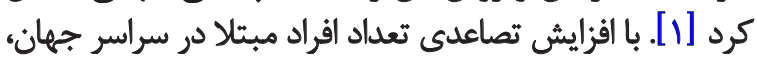

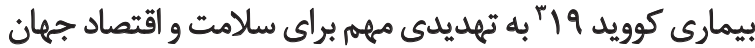

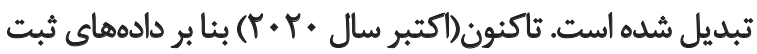

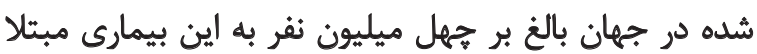

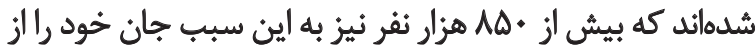
دست دادهاند. در ايران نيز تاكنون بيش از سي هزئ هزار مركى در بين

بحرانهاى شديد، نظير بيمارىهاى همه كير جهانى، بلاياى

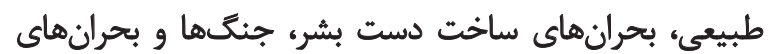

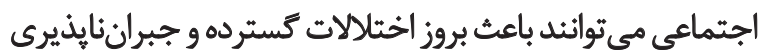

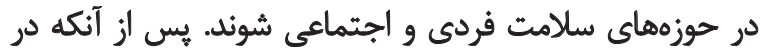

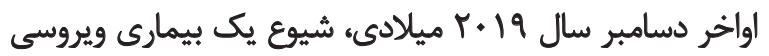

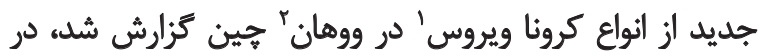

2. Wuhan
\end{abstract}

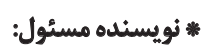

$$
\begin{aligned}
& \text { كتر شيرين جلالى نيانيا }
\end{aligned}
$$

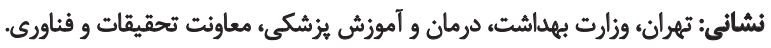

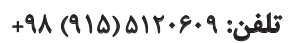

$$
\begin{aligned}
& \text { بست الكثرونيكي: shdjalalinia@gmail.com }
\end{aligned}
$$




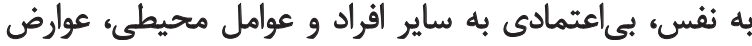

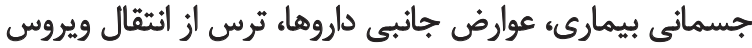

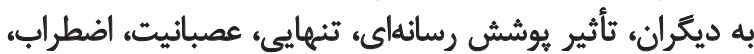

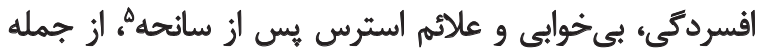

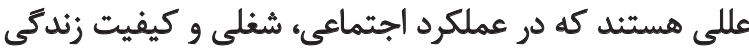
افراد مبتلا يا در معرض خطر اختلال ايجاد مى كنيند [ [ 19]. به رغم اهميث اين مسئله، مطالعات صورت ترفته در اين حوزه

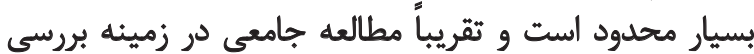

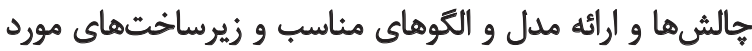

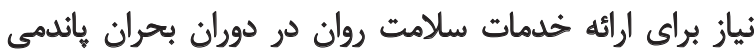

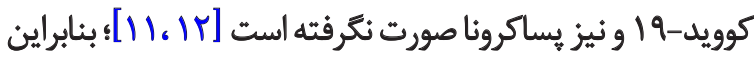

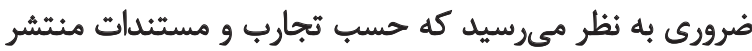

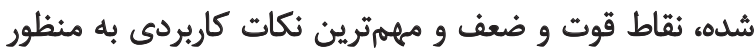

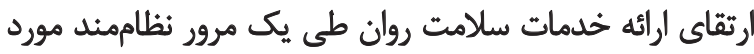

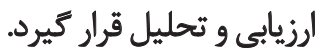

بر اين اساس مطالعه حاضر، با بهرهكيرى از اصول و روشهاى براي

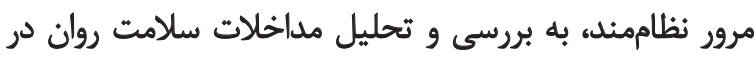

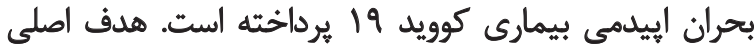

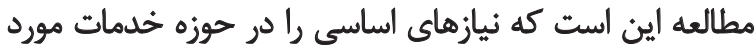

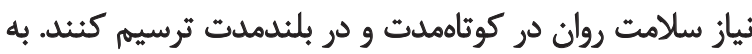

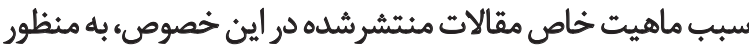

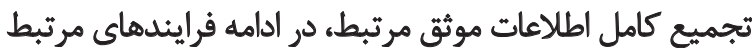

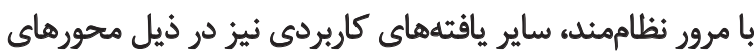

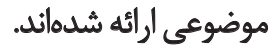

5. Posttraumatic stress symptoms
بالغ بر ششصد هزار نفر مبتلا اتفاق افتاده است كه البته اين آمارها

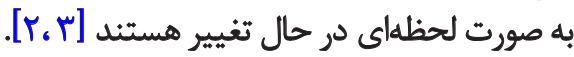
شيوع ناكمانى يك بيمارى همواره تهديدى جدى برائ براي سلامت

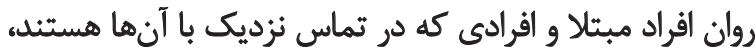

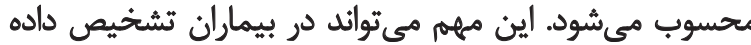

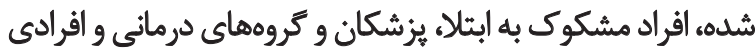

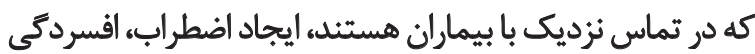

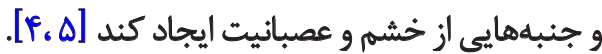

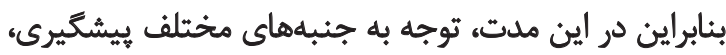

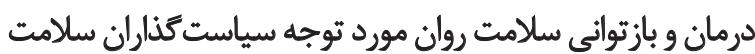

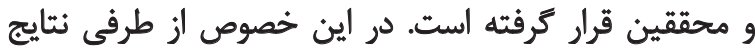

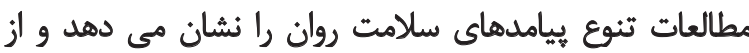

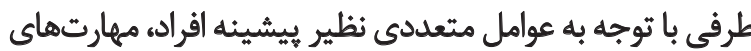

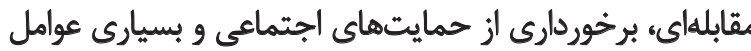

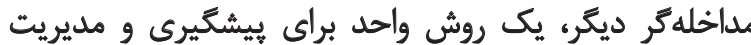

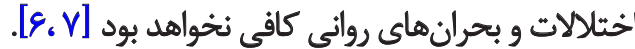
به دليل نوظهور بودن بيمارى، در مورد علائم و اختلالات

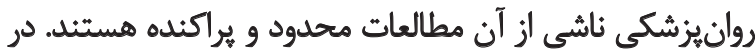

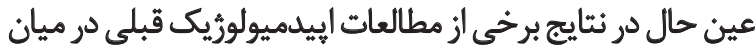

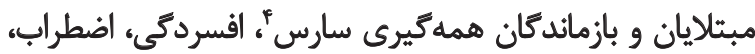

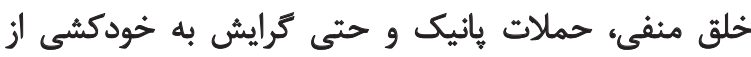

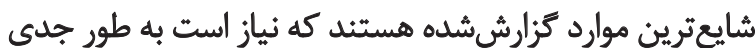

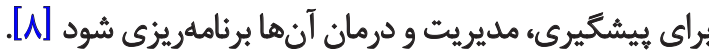
بر اساس مطالعات منتشرشده، انزواى اجتماعى ، كاهش اعتماد

\section{Indexs}

PubMed (covid) OR(coronavirus) AND("Mental Health Services"[Mesh]) OR ("Mental HygieneServices") OR ("Counseling"[Mesh]) OR (“Hotlines" [Mesh]) OR (Hotline OR care [Title/Abstract])

(TITLE-ABS-KEY (covid) OR TITLE-ABS-KEY (coronavirus)

Scopus (AND TITLE-ABS-KEY ("Mental Health Services") OR TITLE-ABS-KEY ("Mental Hygiene Services”) OR TITLE-ABS-KEY (counseling) OR TITLE-ABS-KEY (hotlin*) OR TITLE-ABS-KEY (care)

TOPIC: (Covid) OR TOPIC: (Coronavirus)

Indexes=SCI-EXPANDED, SSCl, A\&HCI, CPCI-S, CPCI-SSH, BKCI-S, BKCI-SSH, ESCI, CCR-EXPANDED, IC Timespan=All years TOPIC: ("Mental Health Services") OR TOPIC: (Mental Hygiene Services) OR TOPIC: (counseling) OR TOPIC: (hotlin*)

ISI/WOS OR TOPIC: (care) Indexes=SCl-EXPANDED, SSCl, A\&HCI, CPCl-S, CPCl-SSH, BKCl-S, BKCl-SSH, ESCl, CCR-EXPANDED, IC Timespan=All years \#2 AND \#1

Indexes=SCl-EXPANDED, SSCI, A\&HCI, CPCl-S, CPCI-SSH, BKCI-S, BKCI-SSH, ESCI, CCR-EXPANDED, IC Timespan=All years 
جدول ז. ارزيابى كيفيت مقالات واردشده به مطالعه

\begin{tabular}{|c|c|c|c|c|c|c|c|c|c|c|}
\hline$\frac{5}{3}$ & 芆 & 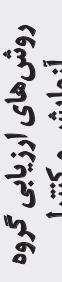 & $\begin{array}{l}\overline{3} \\
\frac{3}{3} \\
\frac{2}{3} \\
\frac{3}{8}\end{array}$ & 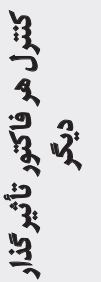 & $\begin{array}{l}\frac{3}{3} \\
\frac{3}{3} \\
\frac{3}{3} \\
\frac{y}{3} \\
\frac{y}{3}\end{array}$ & 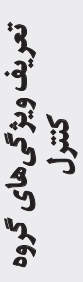 & 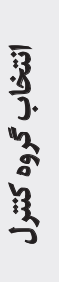 & 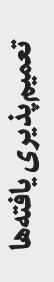 & $\begin{array}{l}2 \\
3 \\
3 \\
3 \\
3 \\
3 \\
3 \\
3 \\
3 \\
3\end{array}$ & مشخصات استناد مطالعه \\
\hline 9 & + & + & + & + & + & + & + & + & + & بو و همكاران [10] \\
\hline$\Delta$ & + & - & + & + & - & - & + & + & - & أكوستينا و همكاران [ع]] \\
\hline$\Delta$ & + & - & + & - & $=$ & - & - & + & + & كئك و همكاران ["r] \\
\hline$\wedge$ & - & + & + & + & + & + & + & + & + & جودسون و همكاران [If] \\
\hline
\end{tabular}

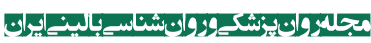

\section{ازوزيابى كيفيت و استخُخراج دادهها}

متعاقب اجراى مراحل جستوجو، نتايج جهت مديريت دادهها

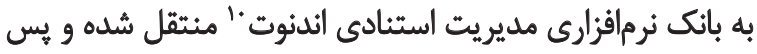

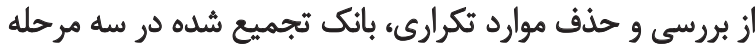

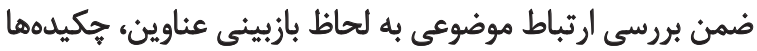

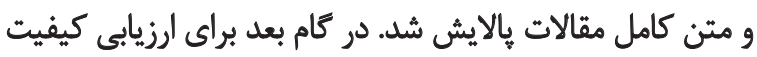

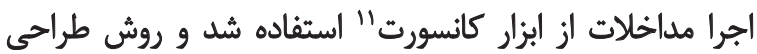

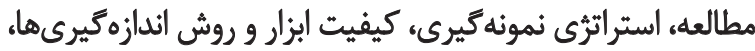

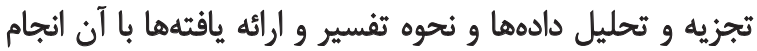

$$
\text { شد (جدول شماره r). }
$$

از موارد حائز شرايط، دادهاي مرتبط با نوع مطالعها، ويرُكى هايى

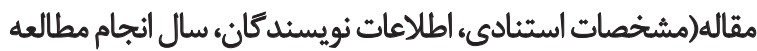

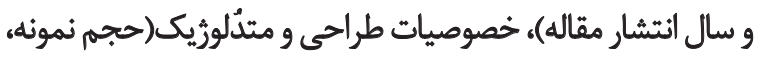

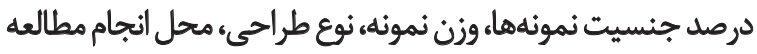

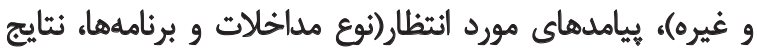

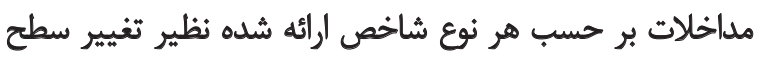

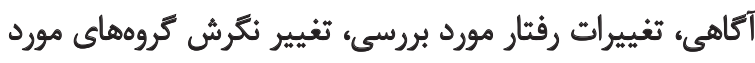

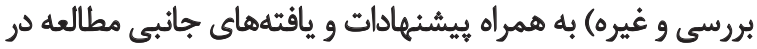

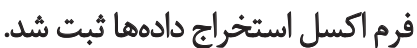

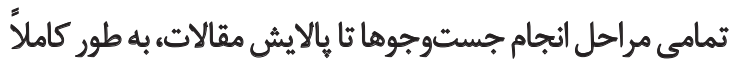

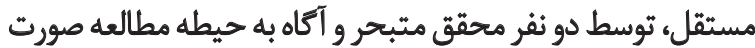

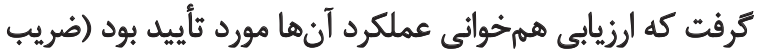
ارزيابى توافق كايا"!

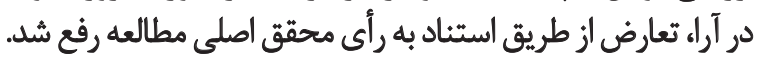

\section{روش - م}

طى اين مرور نظاممند، همه مطالعات مرتبط با بررسى و تحليل

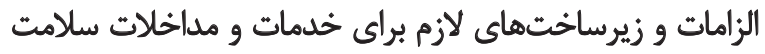

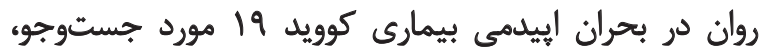

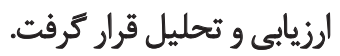

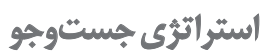

استراترى جستوجو بر مبناى ريشهاى وازثكان كليدى زير نظر Mesh متخصصين مرتبط تدوين شد. با استفاده از اصطلاحئ

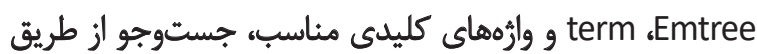

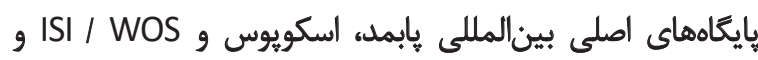

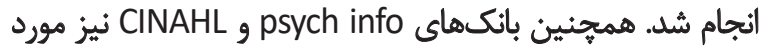
جستوجو قرار كرفت (جدول شماره (1) )

معيارهاى ورود، مقالات جابش بشده در اين مورد مربوط به سال

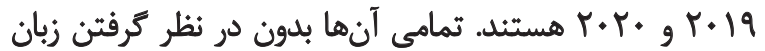

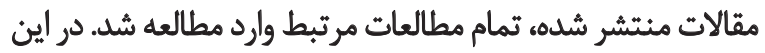

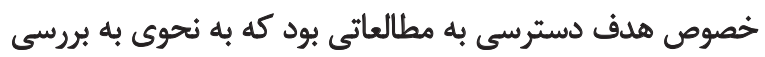

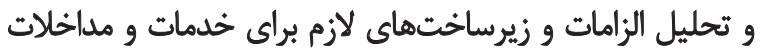
سلامت روان در بحران إيدمى بيمارى كوويد 19 يرداختهانداند. به اين منظور، در صورت موجود بودن در نتايج جستوجوها، لمقات

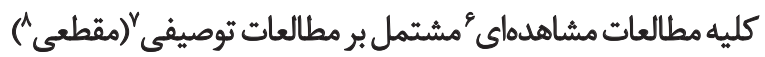

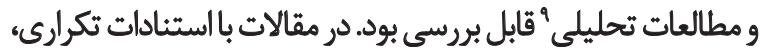

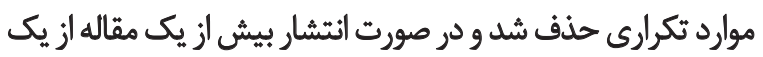
مطالعه واحد، كاملترين منبع داده مورد استفاده قرار كرفت. 
حوزه ياد شده بود. اين يُؤوهشكران تأكيد كردند كه در مقايسه

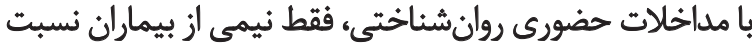

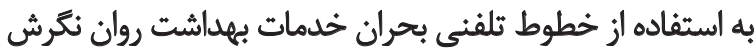

مثبت داشتند [10].

طرح جامع ارائه خدمات مبتنى بر جامعه حسب مناطق برئ

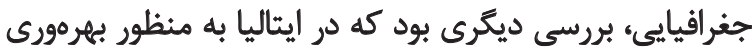

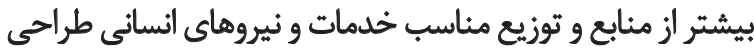

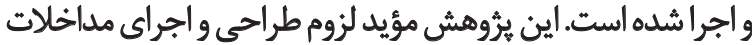

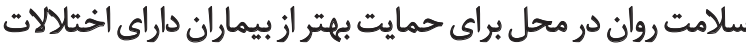

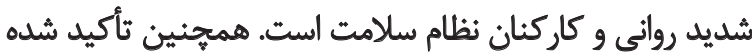

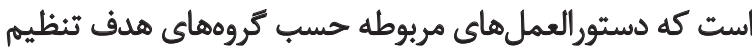

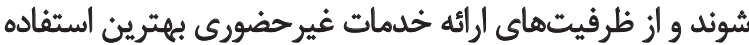

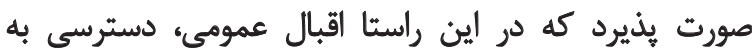

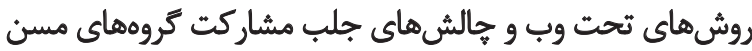

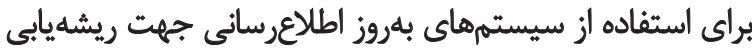

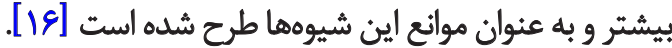

طى مطالعه ديكّرى در جين، افراد در معرض و و مبتلايان به

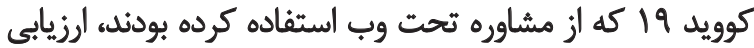

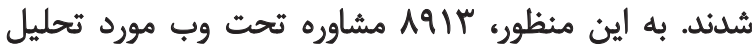

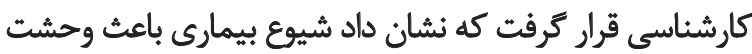

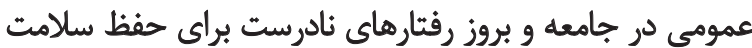

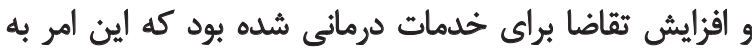

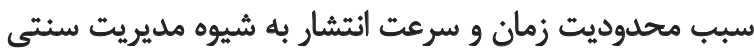

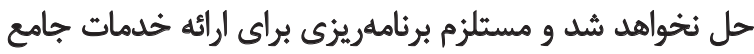

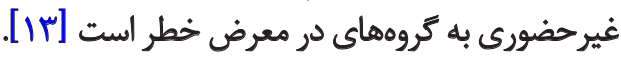

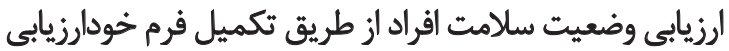

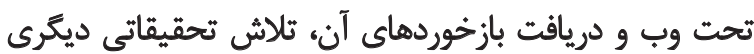

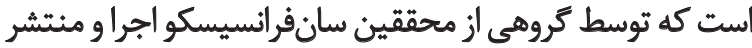

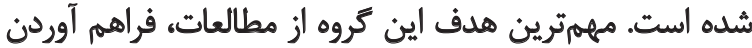

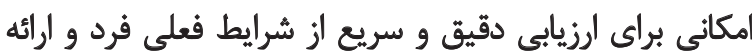

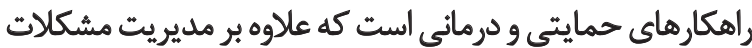

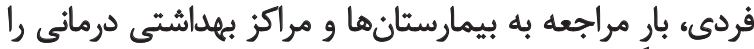

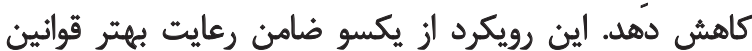

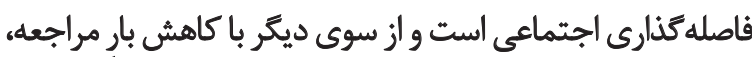

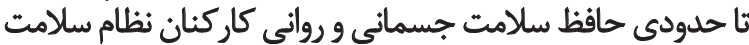

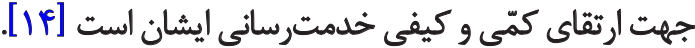

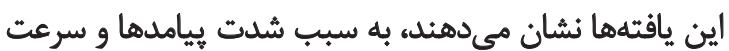

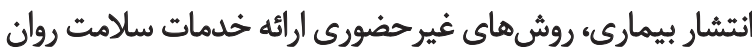

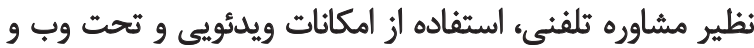

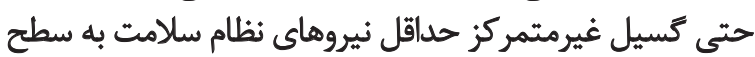

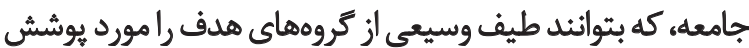
قرار دهند، از راهكار هاي مورد تأكيد محققين هستند. آنجيه اين
يافتهها

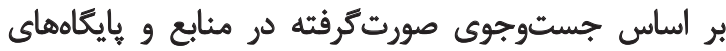

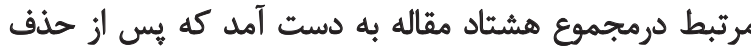

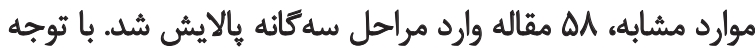

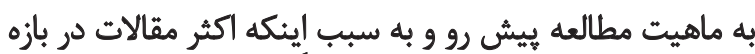

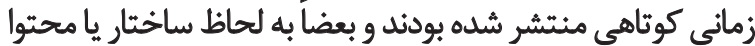

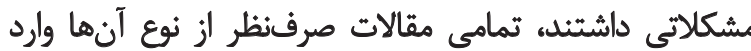

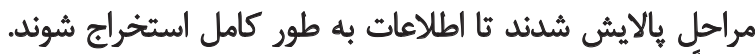

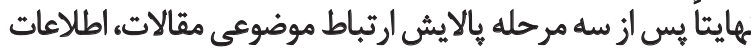

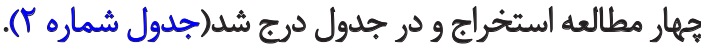

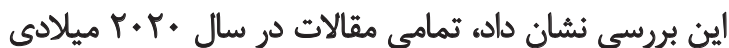

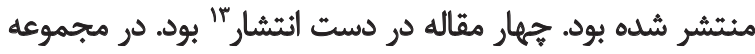

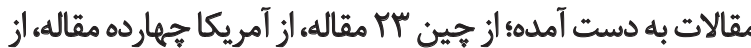

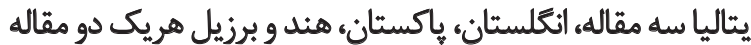

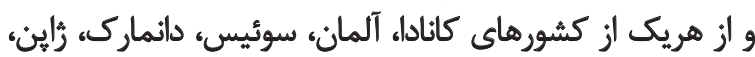

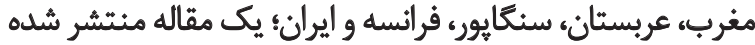

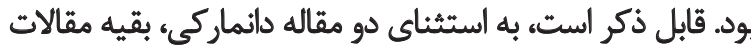

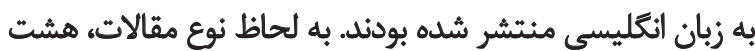

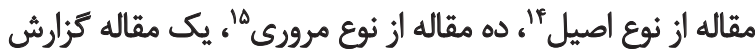

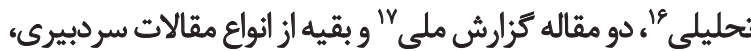

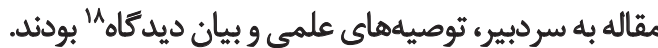
به لحاظ كيفى با توجه به شرايط ويره تأليف و انتشار مقالات

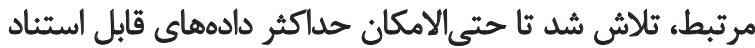

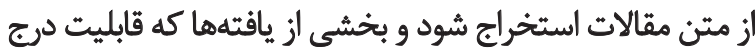

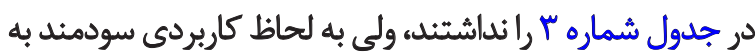

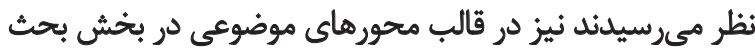

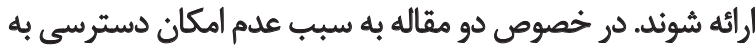

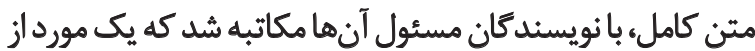

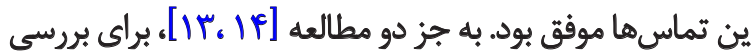

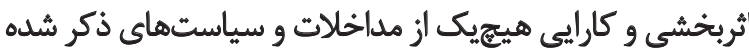

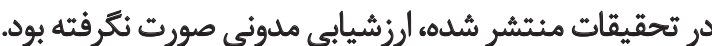

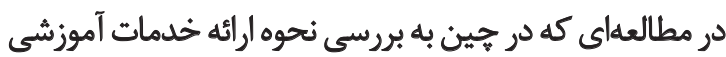
و مشاورهاى از طريق خطوط بحرئ بحران اختصاصى بيمارى كوريد كوريد

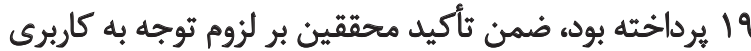

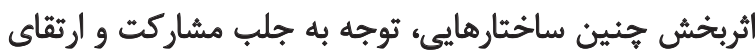

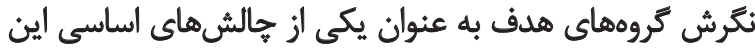

13. In press

14. Original

15. Review

16. Analysis

17. Report

18. Editorial, Letter to the editor, Opinion, commentary, Correspondence 
جدول ऍ. مشخصات و نتايج مطالعات مرتبط
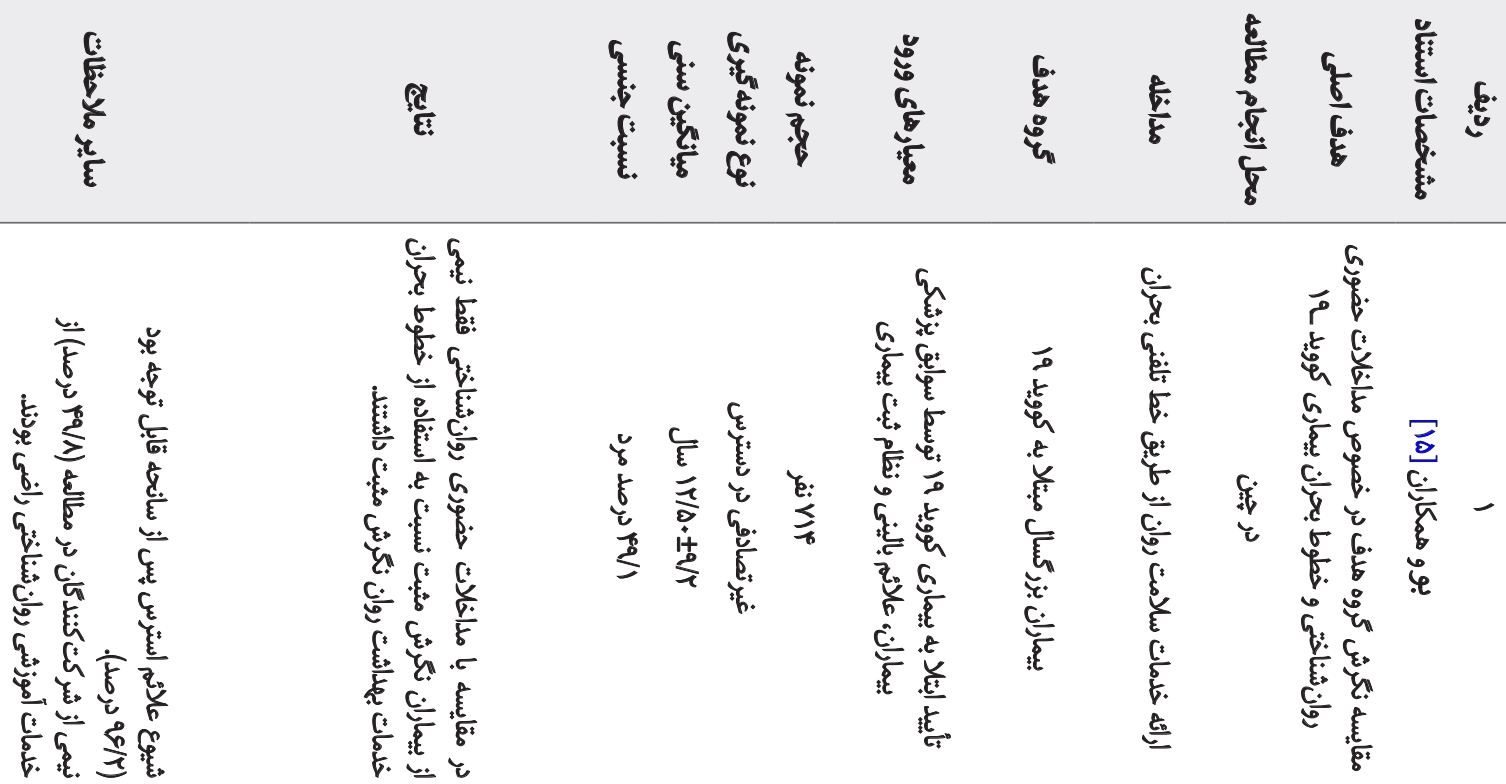

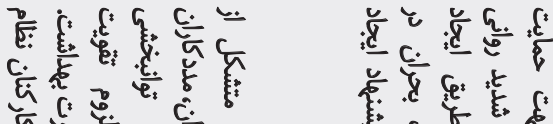

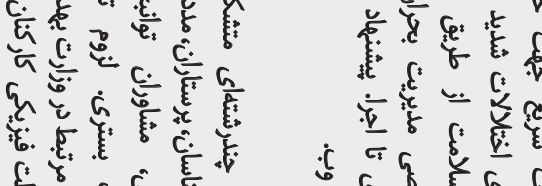

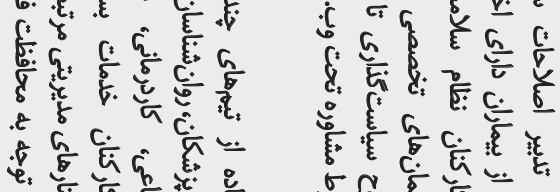

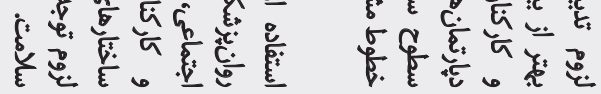

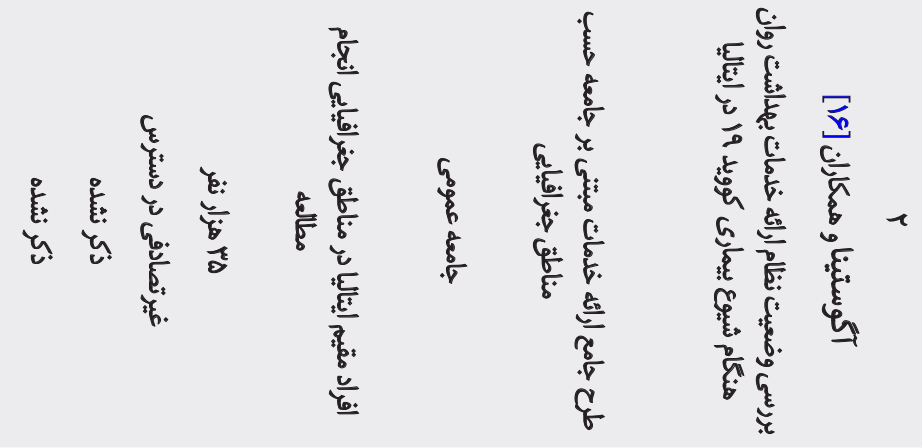

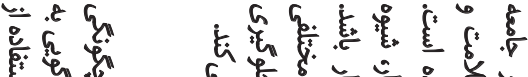

है के है क्ष है है

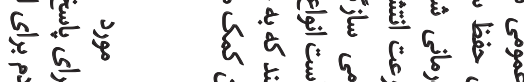

z⿺

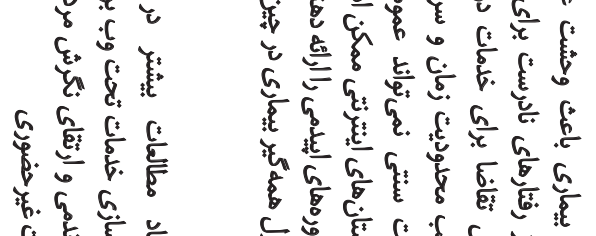

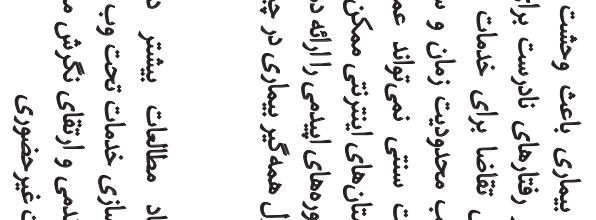

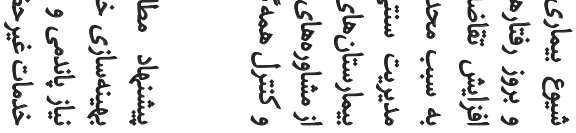

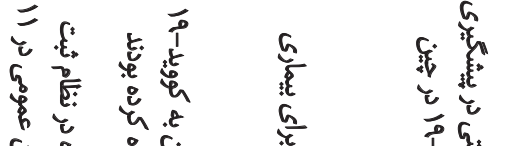

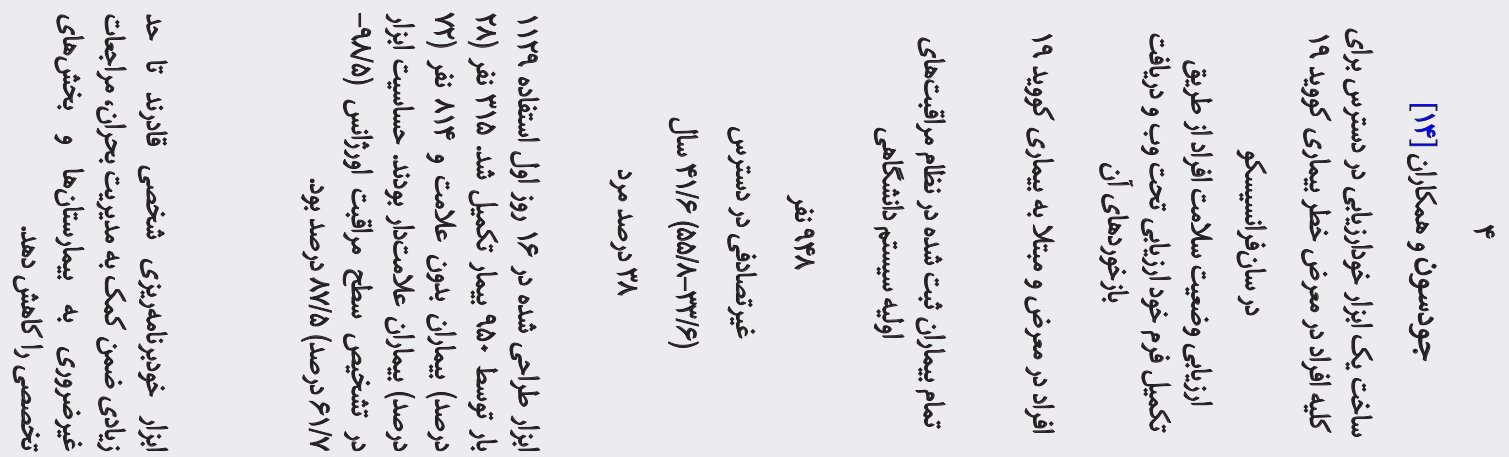


در مطالعات، تمهيد راهكارهايي براي آموزش وسيع جامعه است.

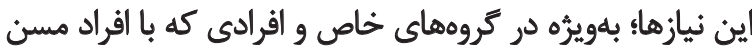

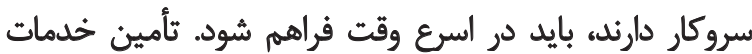

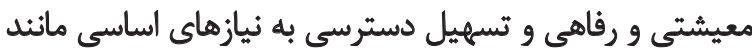

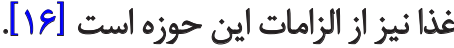

r. سياست كنارى براى افراد و كروههاى در معرض خطر: يكى از

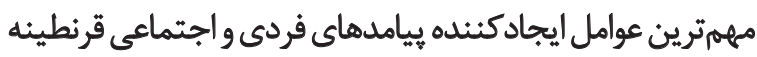

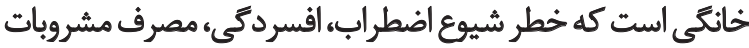

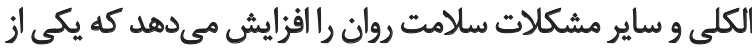

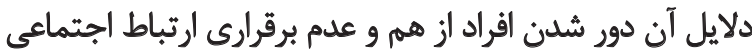

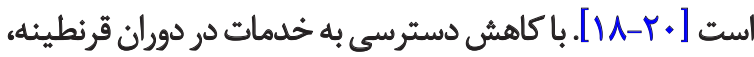

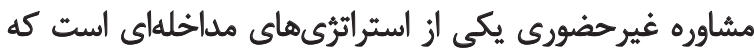

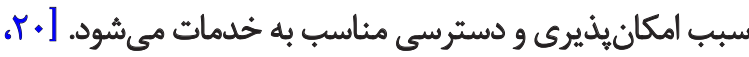

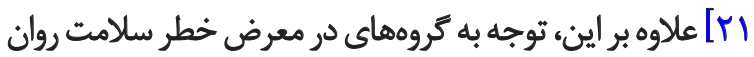

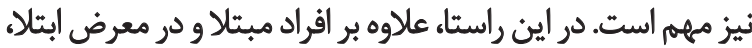

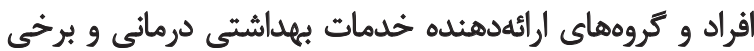

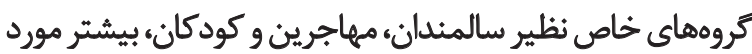

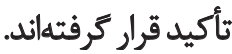

در اين مورد از جمله اقدامات لازم براى آمادهازي بهتر براى

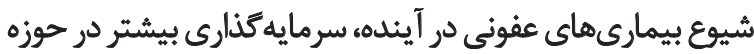

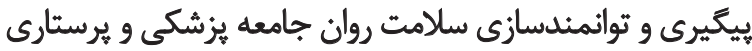
است كه در خط مقدم مبارزه قرار دارند [IV]

در مطالعايى به عنوان راهكار كاهش عوارض روانى كادر

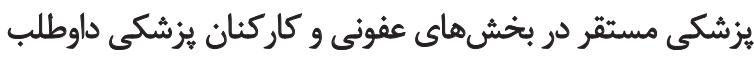

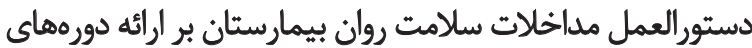

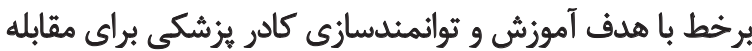

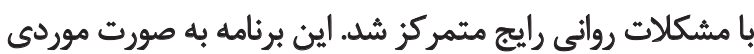

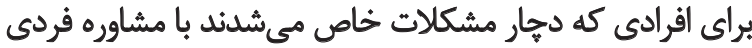

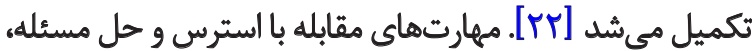

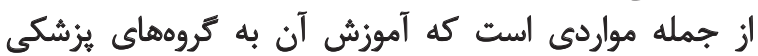

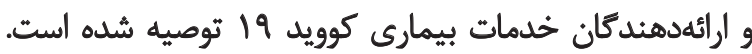

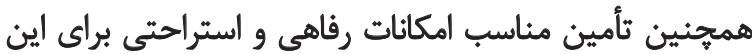

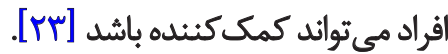

يكى از نكات مورد توجه در خصوص گروههاى در معرض،

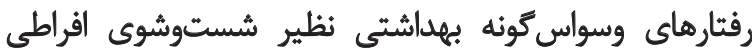

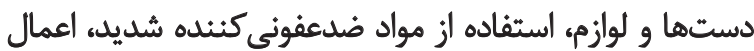

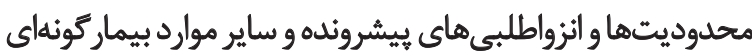

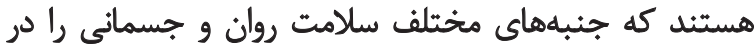

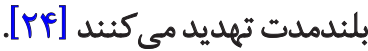

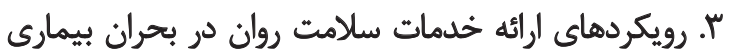

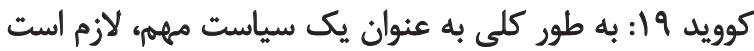
رويكردها به كونهاي اتخاذ شوند كه حداقل تماس بين بين مراجعين

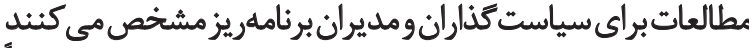

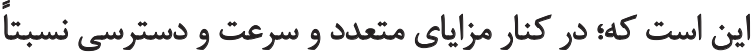

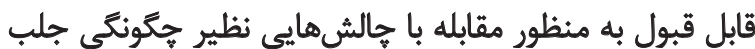

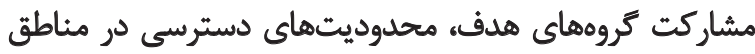

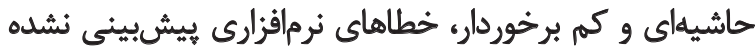

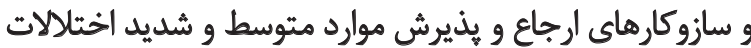

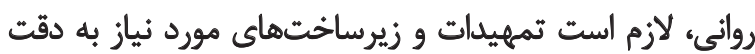

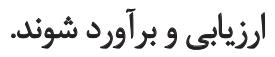
بحث مطالعه حاضر بر اساس طراحى و اجراي الكوى مرور نظاممند

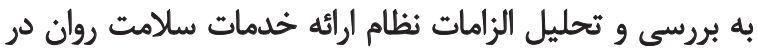
بحران بيمارى كوويد 9 (مي يردازد.

مطالعه ما نشان داد در تمام مقالات با توجه به شدت بئ بيامدها

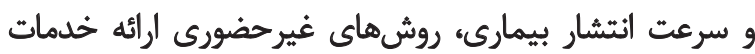

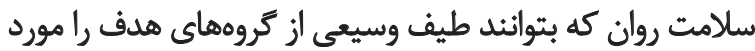

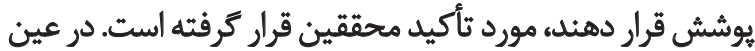

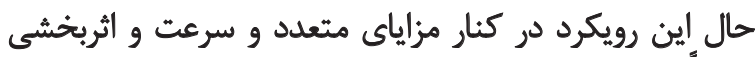

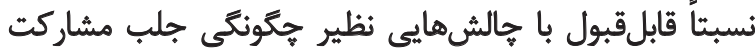

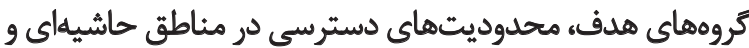

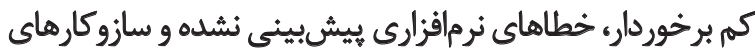
ارجاع و بذيرش موارد متوسط و شرئ شديد اختلالات روانى همراه

همجنين بسيارِى از مطالعاتى كه به بيامدهاى اين بيمارى

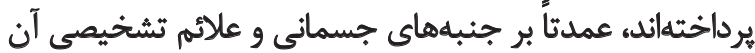

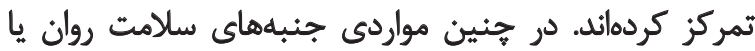

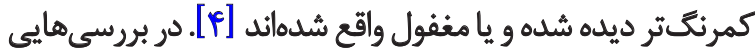

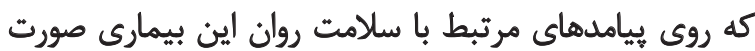

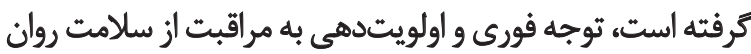

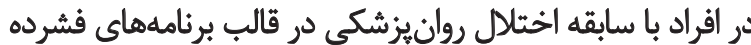

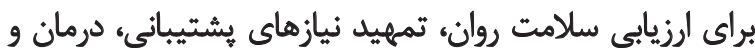

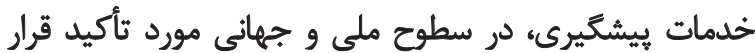

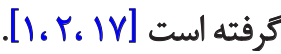

ينج توصيه اساسى در مورد مشكلات روانشئاختى بيمارى

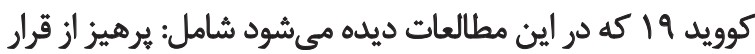

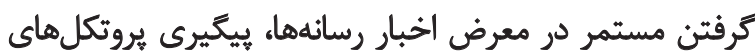

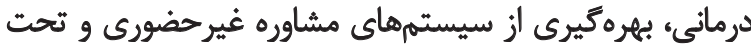

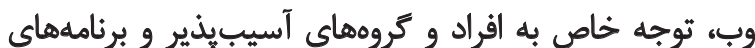

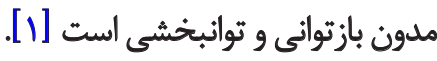

ترمجموع يافتههاى اين مقالات را در زمينههاى زير ميتوان

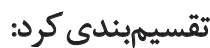

ا. زيرساختها و منابع: ازز مهمترين زيرساختهائ تأكيد شده 


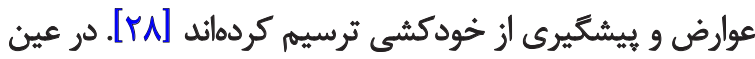

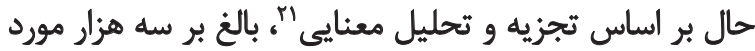

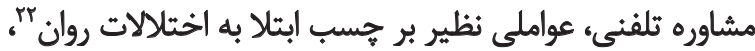

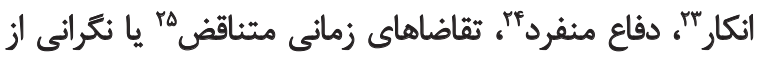

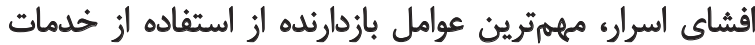
غير حضورى مشاوره بودند [ها]]

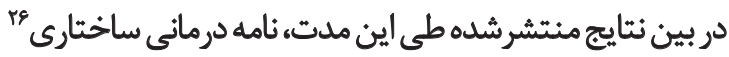

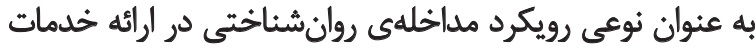

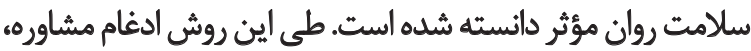

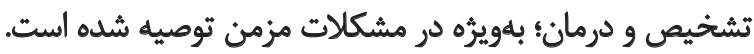

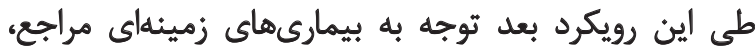

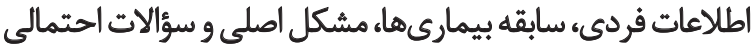

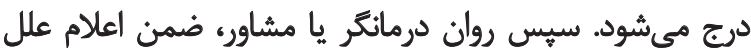

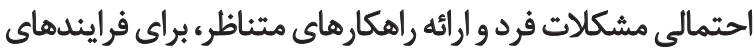

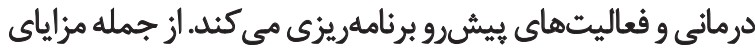

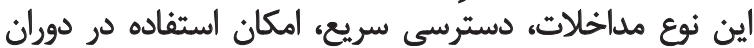

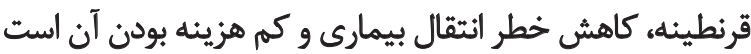

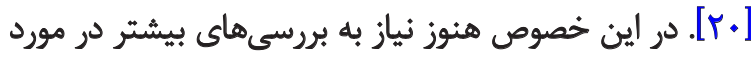

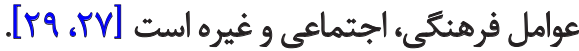

در خصوص استفاده از خدمات برخط، در كنار مزايا، همجينان

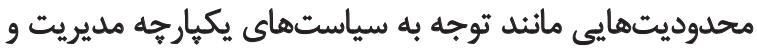

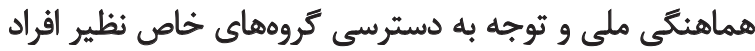

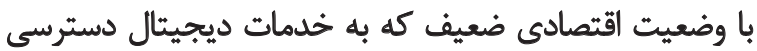

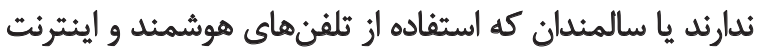

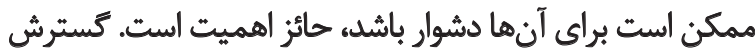

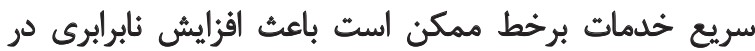

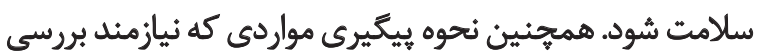

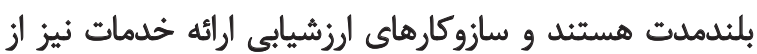

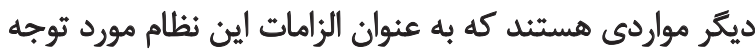

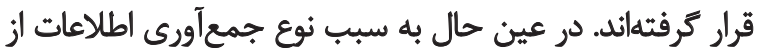

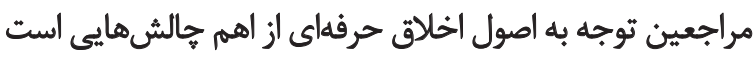

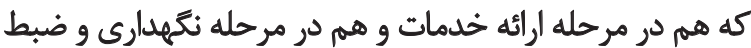

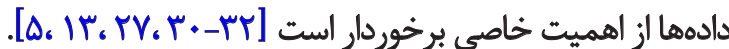
تجربه استراليا در استفاده از خدمات غيرحضورى و مشاوره

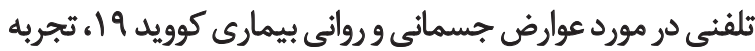
موفقى بوده است كه علاوه بر كاستن از خطر ابتلاي افراد ازئ طريق

21. Semantic analysis

22. Stigma

23. Denial

24. Dissociative defenses

25. Conflicting time demands

26. Coronavirus
و كيرندكان خدمت و ارائهدهندكان خدمات حوزه سلامت روان

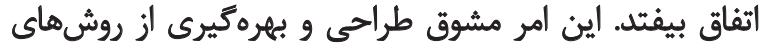

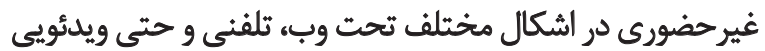

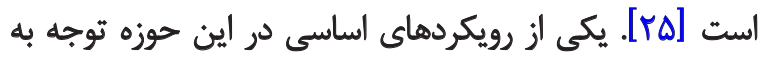

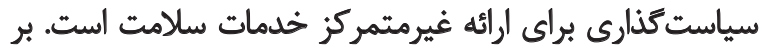

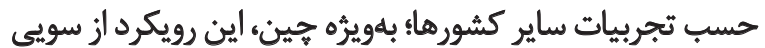

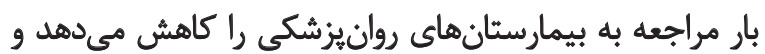

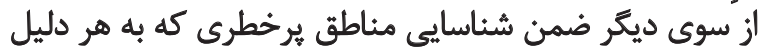

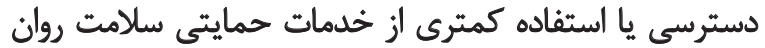

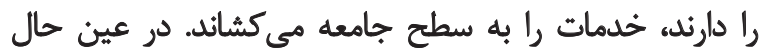

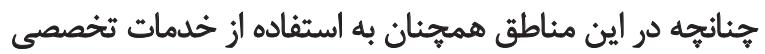

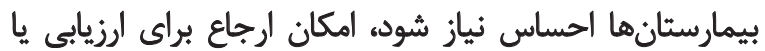
بسترى اين اقراد ديده شده است [9].

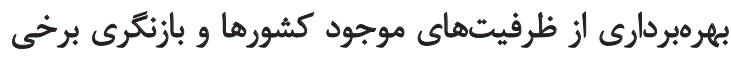

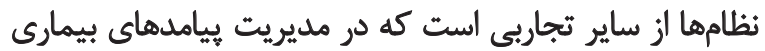

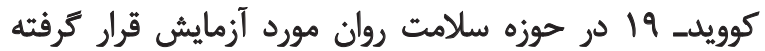

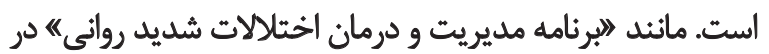

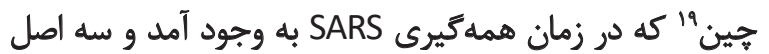

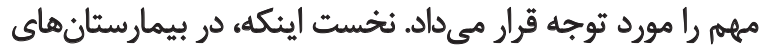

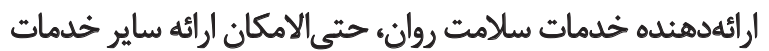

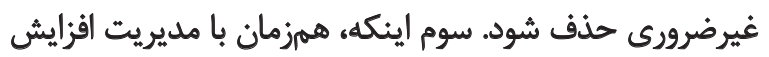

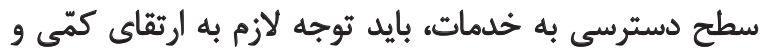

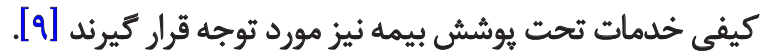

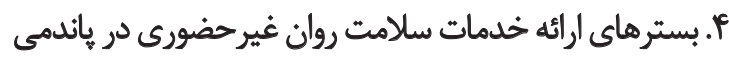

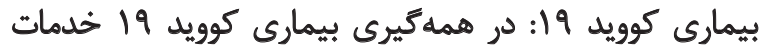

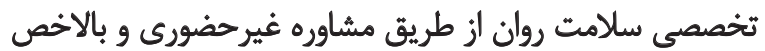

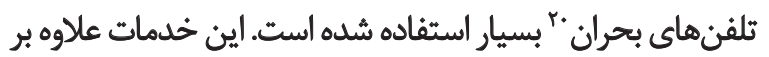

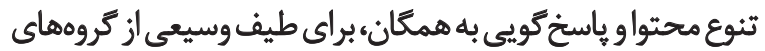

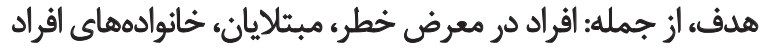

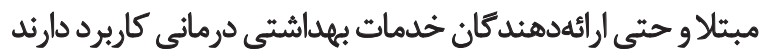

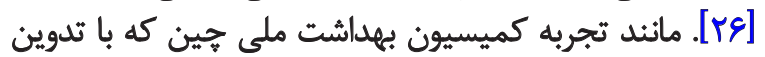

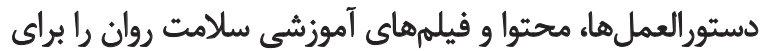

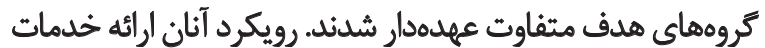

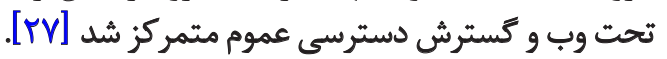

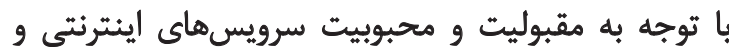
تلفنهاى هوشمند، ضمن استفاده از دستورالعملهايت إنهائ ارئه

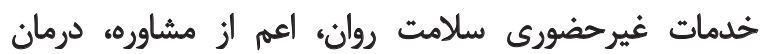

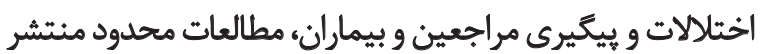

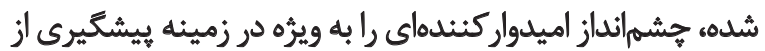

19. Management and treatment program for severe mental illness

20. Tele Mental Health Services 


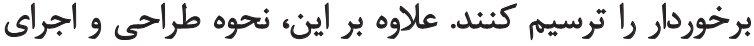

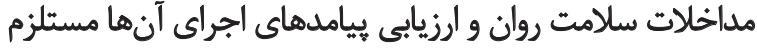

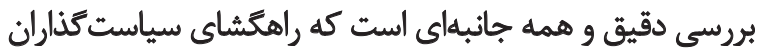

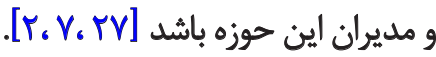

درمجموع به عنوان يكى ار نثاط قوت؛ مطالعه حاضر يك مرور

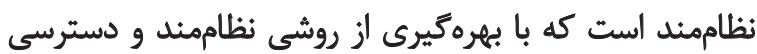

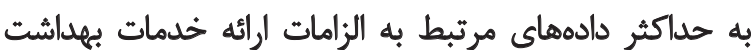

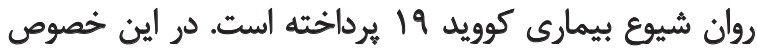

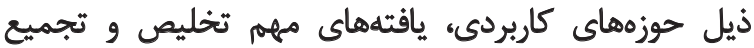

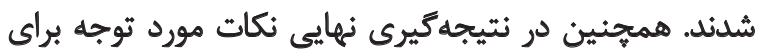

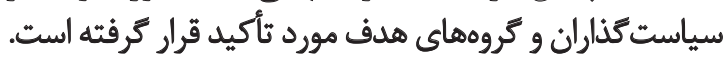

\section{نتيجهيَيرى}

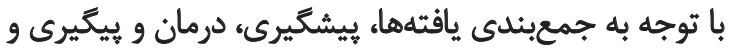

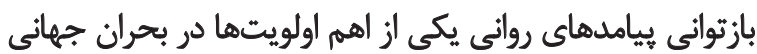

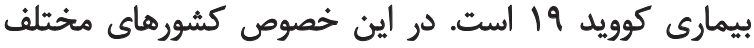

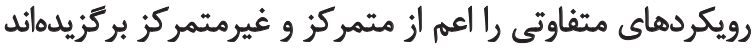

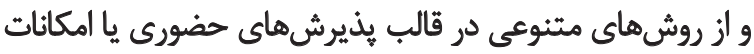

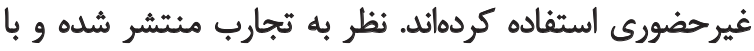

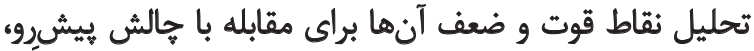

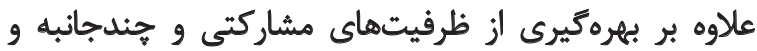

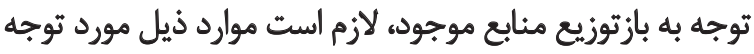
سياست كذاران و مديران حوزههاى سلامت قرار كيرد

ا. جلب مشاركت ساير بخشهاو باو برنامهريزى براى مديريت همه

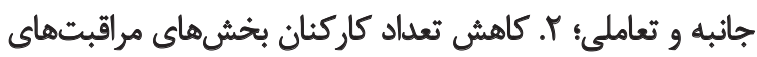

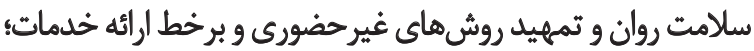

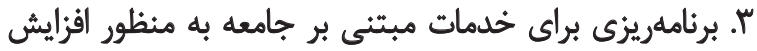

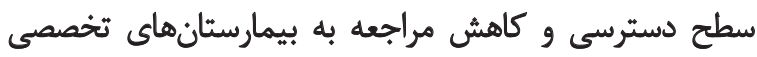

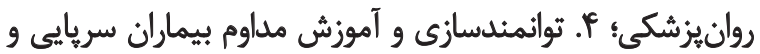
بسترى در بخشهاي روانيزشكى (حضورى و برخط به به اقتضاي

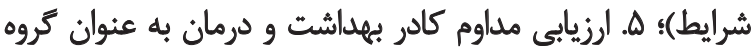

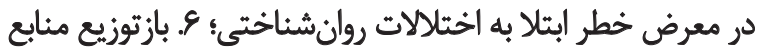

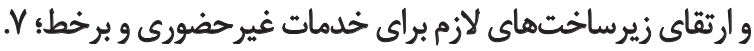

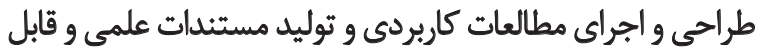

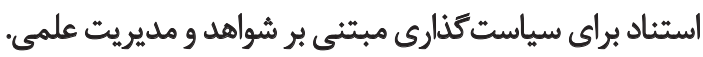

\section{مالاحظات اخلاقى}

از آنجا كه اين مطالعه از نوع مطالعات ثانويه است، ملاحظه

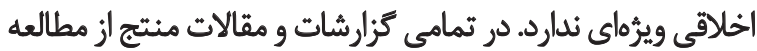
تمامى منبع اطلاعاتى مورد استفاده، استناد خواهد شئد
تماس مستقيم با مراكز درماني و مشاوران، امكان دسترسى سريع إني

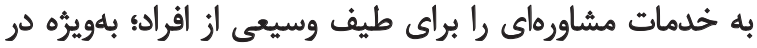

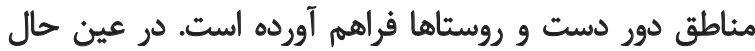

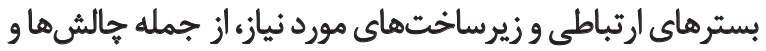

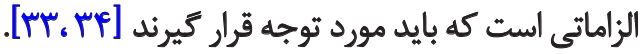
ه. توجهات بِ از درمان و يّيخيرى مبتلايان: برخى مطالعات

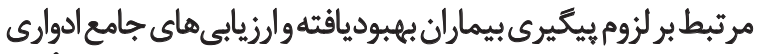

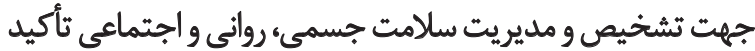

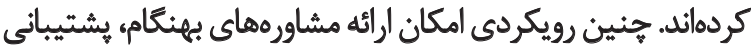

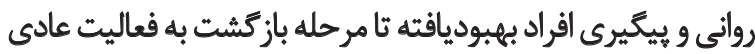

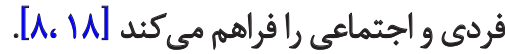

ابتلابه بيمارى كوويد 9 ا و تغيير زندگى روزمره بعد از ترخيص

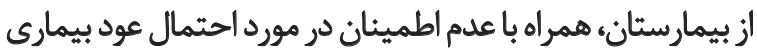

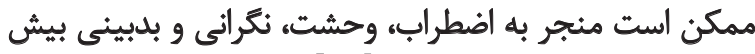

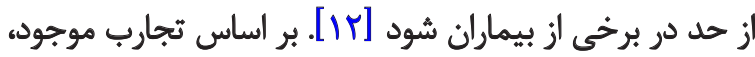

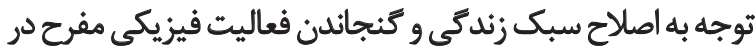

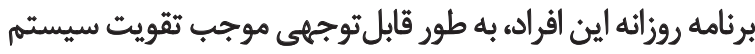

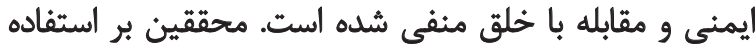

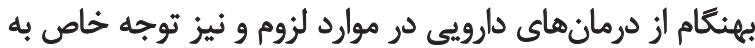

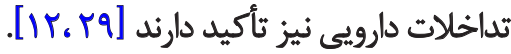

9. زمينههاي تحقيقاتى: همه كيرى بيمارى كوويد 9 امنجر

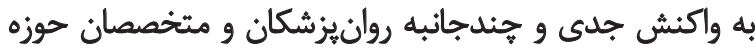

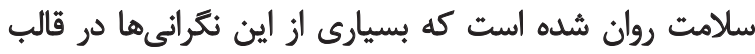

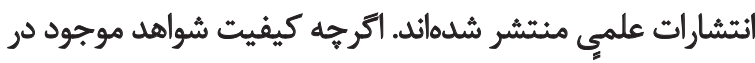

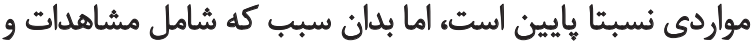

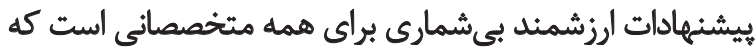

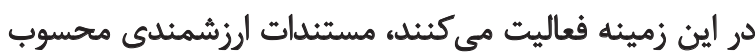

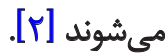

در اين حوزه نقش محققين در تعيين و بيروى اولويتهاي

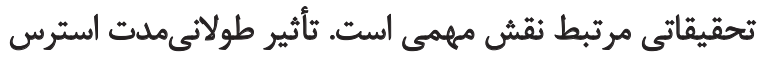

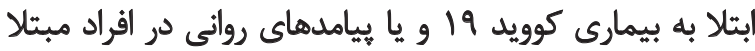

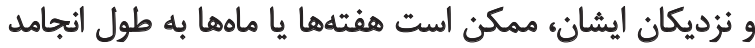

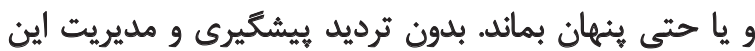

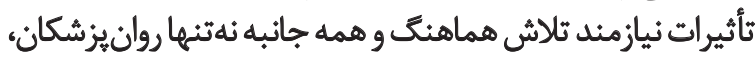

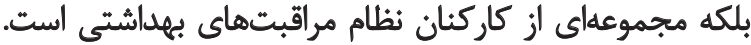

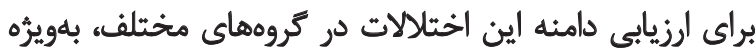

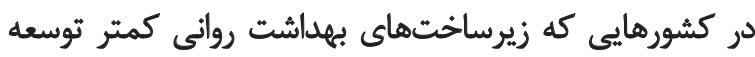

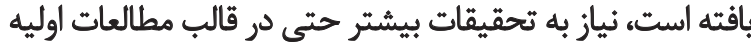

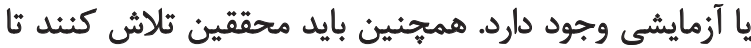

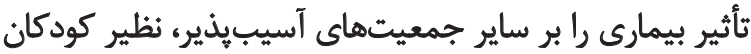

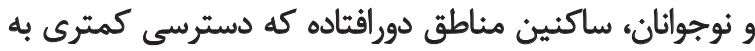

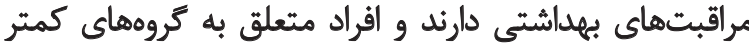




$$
\text { ماهى مالى }
$$

معاونت تحقيقات و فناورى وزارت بهداشت، درمان و آموزش

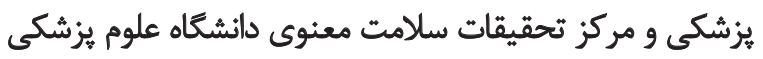
ايران از اين يُروهش حمايت مالى كرده اندي

$$
\text { هشاركث نويسندكان }
$$

تمام نويسندكان در آمادهسازى اين مقاله مشاركت داشتهاند.

$$
\text { تعارض منافع }
$$

بنابر اظهار نويسندكانه اين مقاله تعارض منافع ندارد. 


\section{References}

[1] Ahmed MZ, Ahmed O, Aibao Z, Hanbin S, Siyu L, Ahmad A. Epidemic of COVID-19 in China and associated Psychological Problems. Asian Journal of Psychiatry. 2020; 51:102092. [DOI:10.1016/j.ajp.2020.102092] [PMID] [PMCID]

[2] Rajkumar RP. COVID-19 and mental health: A review of the existing literature. Asian Journal of Psychiatry. 2020; 52:102066. [DOI:10.1016/j.ajp.2020.102066] [PMID] [PMCID]

[3] Singhal T. A review of Coronavirus Disease-2019 (COVID-19). Indian Journal of Pediatrics. 2020; 87(4):281-6 [DOI:10.1007/ s12098-020-03263-6] [PMID] [PMCID]

[4] Huang L, Lin G, Tang L, Yu L, Zhou Z. Special attention to nurses' protection during the COVID-19 epidemic. Critical care (London, England). 2020; 24(1):120. [DOI:10.1186/s13054-020-2841-7] [PMID] [PMCID]

[5] Xiang YT, Zhao YJ, Liu ZH, Li XH, Zhao N, Cheung T, et al. The COVID-19 outbreak and psychiatric hospitals in China: Managing challenges through mental health service reform. International Journal of Biological Sciences. 2020; 16(10):1741-4 [DOI:10.7150/ijbs.45072] [PMID] [PMCID]

[6] Hassan SA, Sheikh FN, Jamal S, Ezeh JK, Akhtar A. Coronavirus (COVID-19): A review of clinical features, diagnosis, and treatment. Cureus. 2020; 12(3):e7355. [DOI:10.7759/cureus.7355]

[7] DePierro J, Lowe S, Katz C. Lessons learned from 9/11: Mental health perspectives on the COVID-19 pandemic. Psychiatry Research. 2020; 288:113024 [DOI:10.1016/j.psychres.2020.113024] [PMID] [PMCID]

[8] Lee AM, Wong JGWS, McAlonan GM, Cheung V, Cheung C, Sham PC, et al. Stress and psychological distress among SARS survivors 1 year after the outbreak. Canadian Journal of Psychiatry. Revue Canadienne De Psychiatrie. 2007; 52(4):233-40. [DOI:10 .1177/070674370705200405] [PMID]

[9] Song Z, Hu Y, Zheng S, Yang L, Zhao R. Hospital pharmacists' pharmaceutical care for hospitalized patients with COVID-19: Recommendations and guidance from clinical experience. Research in social \& administrative pharmacy: RSAP. 2021; 17(1):2027-31. [DOI:10.1016/j.sapharm.2020.03.027] [PMID] [PMM CID]

[10] Liang Y, Chen M, Zheng X, Liu J. Screening for Chinese medical staff mental health by SDS and SAS during the outbreak of COVID-19. Journal of Psychosomatic Research. 2020; 133:110102. [DOI:10.1016/j.jpsychores.2020.110102] [PMID] [PMCID]

[11] Greenberg N, Docherty M, Gnanapragasam S, Wessely S. Managing mental health challenges faced by healthcare workers during covid-19 pandemic. The BMJ(Clinical research ed). 2020; 368:m1211. [DOI:10.1136/bmj.m1211] [PMID]

[12] Cosic K, Popovic S, Sarlija M, Kesedzic I. Impact of human disasters and COVID-19 pandemic on mental health: Potential of digital psychiatry. Psychiatria Danubina. 2020; 32(1):25-31. [DOI:10.24869/psyd.2020.25] [PMID]

[13] Gong K, Xu Z, Cai Z, Chen Y, Wang Z. Internet hospitals help prevent and control the epidemic of covid-19 in China: Multicenter user profiling study. Journal of Medical Internet Research. 2020; 22(4):e18908. [DOI:10.2196/18908] [PMID] [PMCID]

[14] Judson TJ, Odisho AY, Neinstein AB, Chao J, Williams A, Miller $\mathrm{C}$, et al. Rapid Design and Implementation of an Integrated Pa- tient Self-Triage and Self-Scheduling Tool for COVID-19. Journal of the American Medical Informatics Association: JAMIA. 2020 27(6):860-6. [DOI:10.1093/jamia/ocaa051] [PMID] [PMCID]

[15] Bo H-X, Li W, Yang Y, Wang Y, Zhang Q, Cheung T, et al. Posttraumatic stress symptoms and attitude toward crisis mental health services among clinically stable patients with COVID-19 in China. Psychological Medicine. 2020:1-2. [DOI:10.1017/ S0033291720000999] [PMID] [PMCID]

[16] D’Agostino A, Demartini B, Cavallotti S, Gambini O. Mental health services in Italy during the COVID-19 outbreak. The Lance Psychiatry. 2020; 7(5):385-7. [DOI:10.1016/S2215-0366(20)30133-4]

[17] Kang L, Ma S, Chen M, Yang J, Wang Y, Li R, et al. Impact on mental health and perceptions of psychological care among medical and nursing staff in Wuhan during the 2019 novel coronavirus disease outbreak: A cross-sectional study. Brain, Behavior, and Immunity. 2020; 87:11-7. [DOI:10.1016/j.bbi.2020.03.028] [PMID] [PMCID]

[18] Ho CS, Chee CY, Ho RC. Mental Health Strategies to Combat the Psychological Impact of COVID-19 Beyond Paranoia and Panic. Annals of the Academy of Medicine, Singapore. 2020; 49(3):155-60. [DOI:10.47102/annals-acadmedsg.202043]

[19] Navarrete-Reyes AP, Avila-Funes JA. Staying in a burning house: Perks and perils of a hotline in the times of covid-19. Journal of the American Geriatrics Society. 2020; 68(5):E10-1. [DOI:10.1111/igs.16479] [PMID] [PMCID]

[20] Xiao C. A novel approach of consultation on 2019 novel Coronavirus (COVID-19)-related psychological and mental problems: Structured letter therapy. Psychiatry Investigation. 2020 17(2):175-6. [DOI:10.30773/pi.2020.0047] [PMID] [PMCID]

[21] Brownstone ND, Thibodeaux QG, Reddy VD, Myers BA, Chan SY, Bhutani T, et al. Novel Coronavirus Disease (COVID-19) and biologic therapy in psoriasis: Infection risk and patient counseling in uncertain times. Dermatology and Therapy. 2020; 10(3):1-11. [DOI: 10.1007/s13555-020-00377-9] [PMCID]

[22] Chen Q, Liang M, Li Y, Guo J, Fei D, Wang L, et al. Mental health care for medical staff in China during the COVID-19 outbreak. The lancet Psychiatry. 2020; 7(4):e15-6. [DOI:10.1016/ S2215-0366(20)30078-X]

[23] Rana W, Mukhtar S, Mukhtar S. Mental health of medical workers in Pakistan during the pandemic COVID-19 outbreak. Asian Journal of Psychiatry. 2020; 51:102080. [DOI:10.1016/j. ajp.2020.102080] [PMID] [PMCID]

[24] Percudani M, Corradin M, Moreno M, Indelicato A, Vita A Mental health services in lombardy during COVID-19 outbreak. Psychiatry Research. 2020; 288:112980. [DOI:10.1016/j.psyy chres.2020.112980] [PMID] [PMCID]

[25] Shalev D, Shapiro PA. Epidemic psychiatry: The opportunities and challenges of COVID-19. General Hospital Psychiatry. 2020; 64:68-71. [DOI:10.1016/j.genhosppsych.2020.03.009] [PMID] [PMCID]

[26] Zhou X, Snoswell CL, Harding LE, Bambling M, Edirippulige $\mathrm{S}$, Bai $\mathrm{X}$, et al. The role of telehealth in reducing the mental health burden from COVID-19. Telemedicine Journal and E-Health: The Official Journal Of The American Telemedicine Association. 2020 26(4):377-9. [DOI:10.1089/tmj.2020.0068] [PMID]

[27] Li W, Yang Y, Liu ZH, Zhao YJ, Zhang Q, Zhang L, et al. Progression of mental health services during the COVID-19 out- 
break in China. International Journal of Biological Sciences. 2020; 16(10):1732-8. [DOI:10.7150/ijbs.45120] [PMID] [PMCID]

[28] Liu S, Yang L, Zhang C, Xiang Y-T, Liu Z, Hu S, et al. Online mental health services in China during the COVID-19 outbreak. The Lancet Psychiatry. 2020; 7(4):e17-8. [DOI:10.1016/S22150366(20)30077-8]

[29] Bao Y, Sun Y, Meng S, Shi J, Lu L. 2019-nCoV epidemic: Address mental health care to empower society. Lancet (London, England). 2020; 395(10224):e37-8. [DOI:10.1016/S01406736(20)30309-3]

[30] Greenhalgh T, Wherton J, Shaw S, Morrison C. Video consultations for covid-19. The BMJ. 2020; 368:m998. [DOI:10.1136/bmj. m998] [PMID]

[31] Shigemura J, Ursano RJ, Morganstein JC, Kurosawa M, Benedek DM. Public responses to the novel 2019 Coronavirus (2019$\mathrm{nCoV}$ ) in Japan: Mental health consequences and target populations. Psychiatry and Clinical Neurosciences. 2020; 74(4):281-2. [DOI:10.1111/pcn.12988] [PMID] [PMCID]

[32] Yao H, Chen JH, Xu YF. Rethinking online mental health services in China during the COVID-19 epidemic. Asian Journal of Psychiatry. 2020; 50:102015. [DOI:10.1016/j.ajp.2020.102015] [PMID] [PMCID]

[33] Snoswell C, Mehrotra A, Thomas E, Smith K-L, Haydon H Caffery L, et al. Making the most of telehealth in COVID-19 responses, and beyond. Croakey Health Media. 2020. https://espace.library.uq.edu.au/view/UQ:3d6cf9d

[34] Ekeland AG, Bowes A, Flottorp S. Effectiveness of telemedicine: A systematic review of reviews. International Journal of Medical Informatics. 2010; 79(11):736-71. [DOI:10.1016/j.ijmede inf.2010.08.006] [PMID] 